\title{
A Constant-Factor Approximation for the Generalized Cable-Trench Problem*
}

\author{
Marcelo P. L. Benedito ${ }^{1}$, Lehilton L. C. Pedrosa ${ }^{1}$, Hugo K. K. Rosado ${ }^{1 \dagger}$ \\ ${ }^{1}$ Instituto de Computação - UNICAMP \\ Campinas - SP - Brasil \\ $\{$ mplb, lehilton, hugo.rosado\}@ic.unicamp.br
}

\begin{abstract}
In the Cable-Trench Problem (CTP), the objective is to find a rooted spanning tree of a weighted graph that minimizes the length of the tree, scaled by a non-negative factor $\tau$, plus the sum of all shortest-path lengths from the root, scaled by another non-negative factor $\gamma$. This is an intermediate optimization problem between the Single-Destination Shortest Path Problem and the Minimum Spanning Tree Problem. In this extended abstract, we consider the Generalized CTP (GCTP), in which some vertices need not be connected to the root, but may serve as cost-saving merging points; this variant also generalizes the Steiner Tree Problem. We present an 8.599-approximation algorithm for GCTP. Before this paper, no constant approximation for the standard CTP was known.
\end{abstract}

\section{Introduction}

An ubiquitous factor in the decision making of telecommunications and power distributions companies is the cost of deployment of a network infrastructure. This cost might be associated with the deployment of the network, as well as the cost of preparing the location for the deployment. For example, consider the scenario where there is a set of clients which must be connected to a centralized hub through cables. To connect cables between two locations, one must first dig a trench between them. Once the trench is built, any number of cables may be deployed. The Cable-Trench Problem (CTP) aims to minimize the combined cost of digging trenches and deploying cables. This problem was introduced by Vasko et al. (2002) and, although there is a mistake in their NP-hardness proof, this problem can be shown to be NP-hard by a reduction from the 3SAT. It is worth noting that CTP is a natural generalization of Minimum Spanning Tree and Single-Destination Shortest Path, which are classical optimization problems solvable in polynomial time.

In practice, not all locations of a network must be connected, and thus CTP might be too restrictive when clients may be routed through locations serving as merging points. Ignoring such locations might increase the overall connection cost, while including them as clients might incur in unnecessary cable costs. To tackle this situation, we introduce the Generalized Cable-Trench Problem (GCTP), which unlike CTP might contain optional non-client locations.

*This project was supported by São Paulo Research Foundation (FAPESP) grant \#2015/11937-9 and National Council for Scientific and Technological Development (CNPq) grants \#425340/2016-3, \#313026/2017-3, \#422829/2018-8 and \#425806/2018-9.

†Corresponding author. 
Although CTP has been widely studied in the last two decades (Vasko et al., 2002; Nielsen et al., 2008; Rocha, 2018), and many variants have been introduced (Rocha et al., 2017), no constant-factor approximation algorithm is known. In this extended abstract, we focus on the Generalization CTP. We show that an $\alpha$-approximation for CTP can be used as a black box to obtain an approximation algorithm for GCTP with factor $(3+\sqrt{17}) \alpha / 2$. As we also give the first approximation algorithm for CTP with factor $1+\sqrt{2} \approx 2.415$ (Theorem 2 , whose proof will be presented in a full-length paper), this implies the existence of an approximation algorithm for GCTP with factor $(3+\sqrt{17})(1+\sqrt{2}) / 2 \approx 8.599$ (Theorem 6 ).

\section{Definitions and Auxiliary Results}

Let $G=(V, E)$ be a graph and $c: E \rightarrow \mathbb{Q}^{+}$. We denote by $P_{G}(v, u)$ a shortest path from $v$ to $u$ in $G$, and define $c\left(P_{G}(v, u)\right)$ as the sum of the cost of the edges in $P_{G}(v, u)$. When $T$ is a tree rooted at a vertex $r$, we simplify the notation writing $P_{T}(v):=P_{T}(v, r)$. Given a subset $R \subseteq V$ of the vertices, known as terminals, we define the functions $c_{\gamma}(T):=\sum_{v \in R} c\left(P_{T}(v)\right)$ and $c_{\tau}(T):=\sum_{e \in E_{T}} c(e)$. We omit the subscripts when they are clear from the context.

In the Generalized Cable-Trench Problem (GCTP), an instance is a sixtuple $(G, R, r, c, \gamma, \tau)$ composed of a connected graph $G=(V, E)$, a set of terminals $R \subseteq V$, a root $r \in R$, an edge cost function $c: E \rightarrow \mathbb{Q}^{+}$, a cable scaling parameter $\gamma \in \mathbb{Q}^{+}$, and a trench parameter $\tau \in \mathbb{Q}^{+}$. A solution is a tree $S$ that spans $R$ and is rooted at $r$. The objective is to find a solution that minimizes $\mathbb{C}(S):=\tau \cdot c_{\tau}(S)+\gamma \cdot c_{\gamma}(S)$.

We say that an instance $I=(G, R, r, c, \gamma, \tau)$ is metric if $G$ is a complete graph and $c$ satisfies the triangle inequality. In particular, the Metric Generalized Cable-Trench Problem is the restriction of GCTP to metric instances.

Lemma 1. Let $I=(G, R, r, c, \gamma, \tau)$ be an instance of GCTP. If $I$ is not metric, then $I$ can be reduced in polynomial time to a metric instance $\left(G^{\prime}, R, r, c^{\prime}, \gamma, \tau\right)$ preserving the approximation factor.

Proof. Let $\left(G^{\prime}, c^{\prime}\right)$ be the metric closure of $G$ with respect to $c$. Also, let $S^{*}$ be an optimum solution for $I$ and $S^{*}$ be an optimum solution for $I^{\prime}=\left(G^{\prime}, R, r, c^{\prime}, \gamma, \tau\right)$. Observe that $\mathbb{C}^{\prime}\left(S^{\prime *}\right) \leq \mathbb{C}\left(S^{*}\right)$, since any solution for $I$ is a feasible solution for $I^{\prime}$.

For an edge $e \in E_{G^{\prime}}$, let $P(e)$ be the path on $G$ corresponding to $e$. Given an $\alpha$-approximate solution $S^{\prime}$ for $I^{\prime}$, we construct a corresponding solution $S$ for $I$ by taking $S=G[\mathcal{E}]$, where $\mathcal{E}$ is the union of all edges in $P(e)$ for every edge $e \in E_{S^{\prime}}$.

Now observe that $\mathbb{C}(S) \leq \mathbb{C}^{\prime}\left(S^{\prime}\right)$, as the lengths of shortest paths in $S$ and in $S^{\prime}$ are the same, and the full cost of (trench) edges in $S$ can only get cheaper. It follows that

$$
\mathbb{C}(S) \leq \mathbb{C}^{\prime}\left(S^{\prime}\right) \leq \alpha \cdot \mathbb{C}^{\prime}\left(S^{\prime *}\right) \leq \alpha \cdot \mathbb{C}\left(S^{*}\right)
$$

Because of Lemma 1, from now on we assume without loss of generality that instances of GCTP are always metric. CTP is the particular case of GCTP in which $R=V$, so Lemma 1 also applies to CTP. For CTP, we have the following theorem: 
Theorem 2. There is a $(1+\sqrt{2})$-approximation for the Metric CTP.

Observe that GCTP is an extension of CTP in the same way that the Steiner Tree Problem (STP) is an extension of the Minimum Spanning Tree Problem. A solution need not to span every vertex in the graph, only a few are required, while the others serve as optional cost-reduction consolidation points. Notice that STP is the special case of GCTP where $\gamma=0$, hence the same inapproximability result for STP (Chlebík and Chlebíková, 2008) is also valid for GCTP.

Theorem 3. GCTP is NP-hard to approximate within a factor of 96/95.

\section{Approximating the Generalized Cable-Trench Problem}

We start with an auxiliary definition.

Definition 4. Let $T$ be a tree rooted at a vertex $r$. A subgraph $Y \subseteq T$ is said to be fine if $Y$ is connected and $T-E_{Y}$ is composed of a tree which contains $r$ and a set of isolated vertices.

Suppose $T^{\prime}$ is the tree of $T-E_{Y}$ containing the root $r$. Observe that if $Y$ is a fine subtree of $T$, then $Y$ and $T^{\prime}$ share exactly one vertex, which is called the root of $Y$ and is denoted by $r_{Y}$. For a fine subtree $Y$, denote by $R_{Y}$ the set of terminals spanned by $Y$. We also associate the set of terminals $R_{Y}^{\prime}$ as follows: contract $Y$ into a single vertex $u$; if $u$ is a leaf, then let $R_{Y}^{\prime}=R_{Y}$, otherwise, define $R_{Y}^{\prime}=R_{Y} \backslash\left\{r_{Y}\right\}$.

The following lemma states that one can reduce GCTP to CTP, in polynomial time, increasing the approximation by a factor of at most $(3+\sqrt{17}) / 2 \approx 3.562$. The core idea of the proof is to first partition the sets of edges and the set of terminals of an optimal solution by successively finding fine subtrees with bounded number of vertices. Each subtree $Y$ will be associated with a set of terminals $R_{Y}^{\prime}$ and a set of edges $E_{Y}$.

Lemma 5. Let $I=(G, R, r, c, \gamma, \tau)$ be an instance of the metric GCTP such that $\gamma>0$ and $\tau / \gamma \geq 8 /(\sqrt{17}-1)$, and let $I^{\prime}=(G[R], r, c, \gamma, \tau)$ be an instance of the metric CTP. If $S^{*}$ is an optimal solution for $I$ and $T^{*}$ is an optimal solution for $I^{\prime}$, then

$$
\mathbb{C}\left(T^{*}\right) \leq \frac{(3+\sqrt{17})}{2} \cdot \mathbb{C}\left(S^{*}\right)
$$

Proof sketch. Let $d=(\tau / \gamma)(\sqrt{17}-1) / 4$. Next, we say that a fine subtree $Y \subseteq T$ is $d$-fine if $d / 2 \leq\left|R_{Y}^{\prime}\right| \leq d$.

We will break $S^{*}$ into a set $\mathcal{Y}$ of $d$-fine subtrees and a subtree $S^{\prime}$ rooted at $r$ and with $\left|R_{S^{\prime}}\right| \leq d$. Start by letting $\mathcal{Y}=\emptyset$ and by taking $S^{\prime}=S^{*}$. Now, if $\left|R_{S^{\prime}}\right|>d$, find a $d$-fine subtree $Y$ of $S^{\prime}$. Then, add $Y$ to $\mathcal{Y}$ and remove $R_{Y}^{\prime}$ from $S^{\prime}$. We repeat this process iteratively until $\left|R_{S^{\prime}}\right| \leq d$.

In the following, we create a feasible solution $H$ for the reduced instance $I^{\prime}$. Start with a graph $H$ which is a minimum-cost tree spanning only $R_{S^{\prime}}$ and, for each $Y \in \mathcal{Y}$, let $\mathcal{M}_{Y}$ be a minimum-cost tree which spans only $R_{Y}$, and add $\mathcal{M}_{Y}$ to $H$. Also, for each such $Y$, define $y^{*}=\arg \min _{y \in R_{Y}^{\prime}} c(y, r)$, and add the edge $\left\{y^{*}, r\right\}$ to $H$ at the end of each iteration. Since the sets $R_{Y}^{\prime}$ and $R_{S^{\prime}}$ form a partition of $R$, in 
the end of this process, $H$ is a graph whose vertices span exactly $R$, and, therefore, it serves as an upper bound for the cost of an optimum solution for $I^{\prime}$.

We calculate the trench cost of $H$ by bounding the cost of each tree $Y$ and the edges of the form $\left\{y^{*}, r\right\}$. Since $Y$ is a Steiner tree with regard to $R_{Y}$, and as it is well known that a minimum spanning tree is a 2-approximation for STP, we have

$$
c_{\tau}\left(\mathcal{M}_{Y}\right) \leq 2 \cdot c_{\tau}(Y) .
$$

As for the edge $\left\{y^{*}, r\right\}$, we have

$$
c\left(y^{*}, r\right) \leq \frac{2}{d} \cdot\left|R_{Y}^{\prime}\right| \cdot c\left(y^{*}, r\right) \leq \frac{2}{d} \cdot \sum_{y \in R_{Y}^{\prime}} c(y, r) .
$$

The remaining edges of $H$ correspond to $S^{\prime}$.

The total cable cost is bounded as follows: for each $y \in R_{Y}^{\prime}$, we bound the cost to route from $y$ to $y^{*}$, and from $y^{*}$ to the root $r$. By directing the edges of $\mathcal{M}_{Y}$ towards $y^{*}$, one may observe that each edge of $\mathcal{M}_{Y}$ is used at most $\left|R_{Y}^{\prime}\right|$ times in the path from a terminal $y$ to $y^{*}$. We get

$$
\sum_{y \in R_{Y}^{\prime}} c\left(P_{\mathcal{M}_{Y}}\left(y, y^{*}\right)\right) \leq\left|R_{Y}^{\prime}\right| \cdot \sum_{e \in E_{\mathcal{M}_{Y}}} c(e)=\left|R_{Y}^{\prime}\right| \cdot c_{\tau}\left(\mathcal{M}_{Y}\right) \leq d \cdot 2 \cdot c_{\tau}(Y) .
$$

Also, by the minimality of $y^{*}$, one has

$$
\sum_{y \in R_{Y}^{\prime}} c\left(y^{*}, r\right) \leq \sum_{y \in R_{Y}^{\prime}} c(y, r) .
$$

The remaining terminals correspond to $R_{S^{\prime}}$.

The sets $E_{Y}$ and $E_{S^{\prime}}$ partition the edges of $S^{*}$, and the sets $R_{Y}^{\prime}$ and $R_{S^{\prime}}$ partition the vertices of $S^{*}$. By summing all components, one bounds the overall cost of $H$ :

$$
\begin{aligned}
\mathbb{C}(H) & \leq \sum_{Y \in \mathcal{Y} \cup\left\{S^{\prime}\right\}}(2+2 d) \cdot \tau c_{\tau}(Y)+\sum_{Y \in \mathcal{Y} \cup\left\{S^{\prime}\right\}}(2 / d+1) \cdot \sum_{y \in R_{Y}^{\prime}} \gamma \cdot c(y, r) \\
& =\frac{(3+\sqrt{17})}{2}\left(\tau \cdot c_{\tau}\left(S^{*}\right)+\gamma \cdot \sum_{v \in R} c(v, r)\right) \leq \frac{(3+\sqrt{17})}{2} \cdot \mathbb{C}\left(S^{*}\right) .
\end{aligned}
$$

Theorem 6. There is an approximation algorithm for the metric GCTP with factor $(3+\sqrt{17})(1+1 \sqrt{2}) / 2 \approx 8.599$.

\section{References}

Chlebík, M. and Chlebíková, J. (2008). The steiner tree problem on graphs: Inapproximability results. Theoretical Computer Science, 406(3):207 - 214.

Nielsen, R. H., Riaz, M. T., Pedersen, J. M., and Madsen, O. B. (2008). On the potential of using the cable trench problem in planning of ict access networks. In 2008 50th International Symposium ELMAR, volume 2, pages 585-588. IEEE.

Rocha, U., Ramos, N., Melo, L., Benedito, M., Silva, A., Cano, R., Miyazawa, F., and Xavier, E. (2017). Abordagens heurísticas para o p-cabo-trincheira com localização de instalações. In $2^{\circ}$ Encontro de Teoria da Computação (ETC), volume 2. SBC.

Rocha, U. A. C. (2018). Heuristic techniques for large-scale instances of the cabletrench problem. Dissertação de mestrado, Instituto de Computação.

Vasko, F. J., Barbieri, R. S., Rieksts, B. Q., Reitmeyer, K. L., and Stott, K. L. (2002). The cable trench problem: combining the shortest path and minimum spanning tree problems. Computers \&f Operations Research, 29(5):441 - 458. 